\title{
Chronic Periodontitis and Rheumatoid Arthritis - Microbiological Aspects
}

\author{
Elka Popova ${ }^{1}$, Liliya Kavlakova ${ }^{1}$, Mariya \\ Panchovska².
}

1. Department of Periodontology and Oral mucosal diseases, Faculty of Dental Medicine, Medical University - Plovdiv, Bulgaria;

2. Department of Propaedeutics of Internal Diseases, Medical University - Plovdiv, Bulgaria;

\begin{abstract}
An overview of the literature on the role of periodontopathogenic microorganisms and chronic periodontitis in the emergence and development of rheumatoid arthritis is presented. The paper discusses the microbiological and genetic factors, environmental factors and the pathogenesis of the two diseases and presents evidence for the relationship between them. Porphyromonas gingivalis, associated with the severity of chronic periodontitis is the only microorganism documented to produce peptidylarginine deiminase (PAD), involved in the process of citrullination. $P$. gingivalis infection may generate citrullinated peptides, which trigger anti-cyclic citrullinated peptide (anti-CCP) antibodies against synovial lining of the joints. Citrullination by human PADs is important in normal physiology and inflammation. Porphyromonas gingivalis has not been detected in all investigated patients with rheumatoid arthritis and hasn't been associated with the titer of rheumatoid factor / anti-cyclic citrullinated peptide (anti-CCP) antibodies. Probably in the process of citrullination another microorganism that will be shown in future studies takes an active role. The emphasis is placed on Anaeroglobus geminatus, which is associated with the titer of rheumatoid factor / anti-CCP antibodies and detected in all patients studied. The presence of Leptotrichia and Prevotella spp. only in patients with new-onset rheumatoid arthritis is highlighted.
\end{abstract}

Keywords: rheumatoid arthritis, chronic periodontitis, citrullination, peptidylarginine deiminase, anti-CCP antibodies 


\section{Background}

Rheumatoid arthritis (RA) is a systemic inflammatory autoimmune disease, which is characterised by chronic inflammation of the synovial membrane that lines the cavities of joints as well as progressive destruction of the articular cartilage and the underlying bone.

The pathogenesis of RA is associated with genetic and environmental factors, sustainable activity of the innate and adaptive immunity, and broken immune tolerance (1).

The heterogeneity of pathogenetic mechanisms of RA is associated with the cellular, molecular and genetic levels; they are responsible for the variety of clinical and immunologic manifestations.

The link between chronic periodontitis (CP), oral microorganisms and the immunologic reactions that occur and are responsible for the predisposition to developing RA hasn't been completely clarified (2). Remodelled immune defence against oral microorganisms, altered subgingival environment and a more severe clinical course of existing CP appear in patients with RA $(3,4)$. RA and CP are both chronic inflammatory diseases. CP occurs due to an opportunistic infection (5). Infectious agents are thought to have a role in RA development. Microorganisms are required, of course, but they alone are not sufficient to cause a disease (6).

Porphyromonas species with certain virulence (i.e. invasiveness with high activity of the enzyme Peptidylarginine deiminase (PAD)) can be considered triggering factors for the development of RA in susceptible individuals. It is Porphyromonas gingivalis (P. gingivalis) that is one of the microorganisms in the red complex periodontopathogens and is related to the severity of CP and may be one of the RA causes.

There is a distinct evidence-based similarity that is found in the pathogenesis of both diseases - animal models of RA which develop periodontitis (7). IL-1, TNF- $\alpha$, and PG E2 take part in their pathogenesis. The immune regulation and the balance between Th1 and Th2 are disturbed in RA and CP, including environmental factors. Certain interleukins (ILs) (i.e. IL-1, IL-6, IL-8, IL-15 и IL-17), as well as NF-kappaB ligand (RANKL), take part in the development of RA. The same proinflammatory cytokines participate in the inflammation in CP (8).

Patients with RA show prevalence of CP 8 times higher $(9,10)$. It has been estimated that moderate and severe forms of CP predominate in more than $75 \%$ of the patients with RA in comparison to the control group (6).

From a historical point of view, the link between RA and periodontal disease (PD) dates back to the beginning of the 19th century, when Benjamin Rush found out that total extraction of teeth is a cure for "Rheuma" (11). The treatment by extracting teeth is a method that was used at the beginning of the 20th century. It continued to exist as a procedure in many countries until Cecil et al. came to the conclusion in 1930 that tooth extraction is not a radical solution to the problem concerning successful RA treatment (12).

\section{Rheumatoid arthritis}




\section{Definition}

$\mathrm{RA}$ is the most common immune-mediated inflammatory rheumatic disease that affects up to $1 \%$ of the population. The economic losses for society are incomparable to those with Coronary heart disease (CHD). At the beginning of the 21th century along with the early diagnosis and treatment of immune inflammatory rheumatic diseases, the relationship between RA and the role of different types of microorganisms participating in the pathogenesis of the disease has been a subject of study (13).

\section{Etiology}

\section{- Link between rheumatoid arthritis and microorganisms:}

The etiological factors causing RA are unknown. The disease occurs in genetically predisposed individuals due to the influence of environmental factors (1).

The role of various bacteria and viruses has been a subject of debate: T-cell lymphotropic virus type 1, the Epstein-Barr virus, herpesviruses, Rubella virus, parvoviruses. As for the bacteria, mycoplasmas, mycobacteria, intestinal bacteria - E.coli, etc. are thought to have a role. It has been proved that inserting cell wall fragments of Str.pyogenes and E.coli into laboratory mice can cause alteration of their articular cartilage like rheumatoid inflammation (8).

Bacterial membranes, toxins and other components of microorganisms are able to induce chronic articular inflammation.

The relationship between infectious agents, genetic makeup and immune response are significant in the etiology of RA (14).

\section{- Link between rheumatoid arthritis and genetic factors:}

Genetic factors play a large role in the development of the disease. The majority of patients with RA are carriers of the DR1, DR3, and DR4 alleles. As the class II molecules are linked to the antigen presenting to the CD4 positive T-cells, DR-polypeptides determine the nature of the immune response (8).

The changes in gene expression, which are different from the ones in the primary DNA sequence, are the subject of epigenetics (15). Epigenetic changes appear without a direct change in the genetic sequence and can be reversible. It is thought that they are associated with both RA and CP $(16,17)$.

Superantigenes and heat shock proteins (HSPs) are thermostable, hydrophilic molecules of weight that ranges from 24 to $30 \mathrm{kDa}$. Heat shock proteins (HSPs) are a protein family, produced by cells in response to a stress signal. HSPs take part in the hereditary and the acquired immunity and are related to the pathogenesis of RA, participating in the citrullination processes $(18,19)$.

High levels of oral bacterial HSPs have been found in the serum of patients with RA (Prevotella melaninogenica HSP and P. intermedia HSP). The same HSPs have been found in patients with periodontal diseases. This is another proof of the possible mutual pathogenesis of both diseases $(6,20)$. 


\section{Chronic periodontitis}

\section{Definition and prevalence}

$\mathrm{CP}$ is an inflammatory destructive disease, in which the normal balance between the microbial plaque (bacterial biofilm) and the host response is disrupted. This disruption may be due to changes in the composition of the bacterial biofilm, socioeconomic status, psychoemotional stress, systemic diseases, environmental factors, etc. CP is defined as an infectious disease, which results in inflammation of the tissues supporting a tooth, progressive periodontal attachment and bone loss. It can be diagnosed clinically by observing chronic inflammatory changes in the marginal gingiva, bleeding on probing, clinical attachment loss and formation of a periodontal pocket (5). Although the highest rate of incidence is in adults, it can occur in children and adolescents in response to the direct specific effects of subgingival plaque formation. It is mainly seen as a slowly progressive disease. In the presence of systemic or environmental factors that can modify the host response to the accumulated plaque (such as diabetes, smoking or stress), the progression of the disease can get more aggressive.

\section{Etiology}

Chronic gingivitis is a precursor of $\mathrm{CP}$ and as it progresses it affects the remaining structures of the periodontium (5). The progression from gingivitis to periodontitis is still unknown (8).

Gingivitis develops as a result of non-specific bacterial effect, but specific periodontopathogens are also required; some of them are: orphyromonas gingivalis, Fusobacterium nucleatum, Tannerell forsythia, Treponema denticola, Prevotella intermedia (8). The advanced stages of CP are presented mainly by the genus Porphyromonas, Tannerell and Treponema. They all participate in its pathogenesis.

Microorganisms are required, but they alone are not sufficient to cause chronic inflammatory processes. $\mathrm{P}$. gingivalis is associated with the severity of $\mathrm{CP}$ and cannot be isolated from an intact periodontium. Research shows significant genetic impact on the antibiotic sensitivity in patients with CP (21). Genetic factors trigger immune reactions as a whole. Therefore, there's no doubt that susceptibility to periodontal diseases may be due to genetic mechanisms (22).

Despite this, no unique pathogen has been found yet corresponding to the postulate modified by HenleKoch (21). Partly, the criteria have been met due to the fact that it's possible for periodontium to be infected by P.gingivalis (17) and immunisation against this pathogen prevents the development experimental periodontitis in the same animal model $(17,23)$. The purified "porphypains" from P.gingivalis are used as a vaccine providing prevention from periodontitis in animal model $(10,4)$.

\section{Review Results}

\section{- Link between rheumatoid arthritis and chronic periodontitis - the role of bacteria}

The link between the oral microorganisms, CP and RA has been examined in a number of experiments. It is assumed that the oral microorganisms can trigger RA based on serologic methods $(24,25)$. 
The hypothesis for the role of oral infection in the pathogenesis of RA is supported by the study of bacterial DNA of anaerobic organisms and the high titer of antibodies against bacteria in the serum and synovial fluid in patients with RA $(16,26)$. The P.gingivalis periodontopathogen destroys the epithelium and invades the human endothelial cells, thus influencing the transcription and protein synthesis (27).

P.gingivalis can also invade primary human chondrocytes, which have been isolated from knee joints and then induce cell effects. As a consequence of this invasion P.gingivalis leads to apoptosis (28). Many of the bacterial species in the oral cavity, considered to be potential pathogens, can be found in other tissues too $(29,30)$.

In 2004, Rosenstein (23) suggests that P.gingivalis is the only microorganism proved to express peptidylarginine deiminase (PAD) and permits individuals with CP to be exposed to citrullinated antigens. The citrullinated antibodies that are consequently produced predispose to RA development. Citrullination or deamination is responsible for the transformation of arginine amino acid into citrulline amino acid under the enzyme activity of PAD (31). The expression of citrullinated antigens in the synovial fluid proves the role of citrullination in the pathogenesis of RA $(16,32)$ (Figure 1 ).

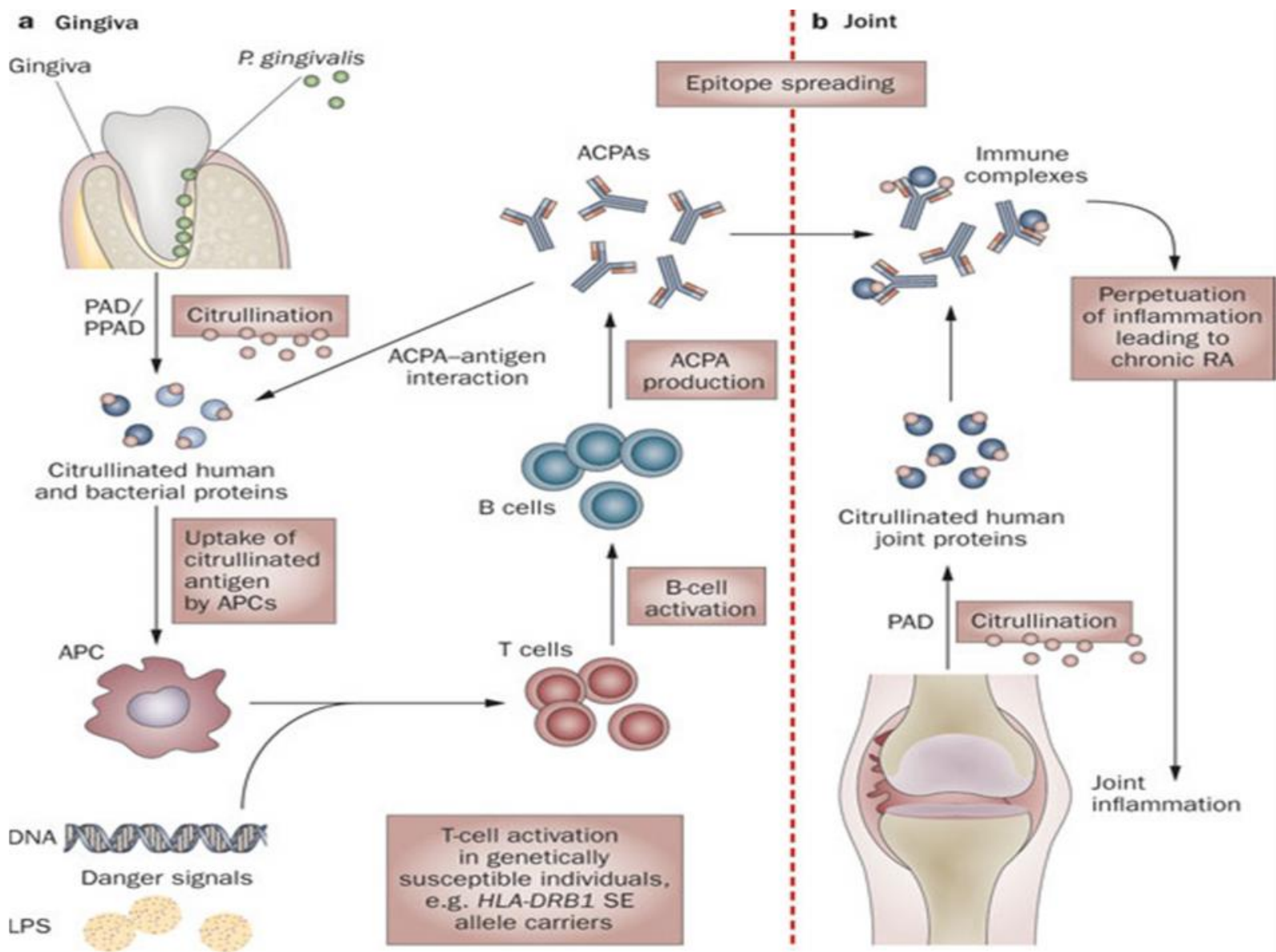

Figure 1. (By Lundberg et al., "Periodontitis in RA - the citrullinated enolase connection", 2010) 
Infection with P. gingivalis leads to citrullination in the gingiva and joints of bacterial and/or human proteins via Peptidylarginine deiminase (PAD). The citrullinated peptides are ingested by the antigen-presenting cells (APCs) in genetically predisposed individuals and the presence of danger signals such as lipopolysaccharides (LPS). Activation of T- and B-cells, differentiation of B-cells into plasma cells and production of specific anti-citrullinated peptide antibodies (ACPA) follow. An antigen-antibody complex is formed and the complement system is activated. A complex cascade reaction is initiated that results in inflammation mediators liberation (27).

It has been estimated that the levels of anti-citrullinated peptide antibody (ACPA) are significantly higher in patients with RA and advanced CP than those in patients with RA without CP. Studies show that the main parodontopathogens in CP are associated with RA (12). Control over periodontal infections can reduce the severity of RA (33). The presence of $P$. gingivalis in patients with RA without CP cannot be explained. Another microorganism with PAD activity is believed to participate in the citrullination, which will be proved in some further investigations $(5,34)$.

Presence of pathogens (PCR-based techniques) associated with CP has been found in a study, consisting of 19 patients who remained after the screening of 500 patients with refractory RA, which is not influenced by disease-modifying antirehumatic drugs (DMARDs). The study estimated that such pathogens were found in $100 \%$ of the bacterial samples taken from knee joints (22). Prevotella intermedia, Treponema denticola and gingivalis are among the most common ones. Aggregatibacter actinomycetemcomitans is the least common one to be found $(31,32)$.

\section{- Chronic periodontitis and rheumatoid arthritis - mutual pathogenesis}

TNF- $\alpha$ and IL-1 play a key role in the pathogenesis of RA. TNF- $\alpha$ regulates the production of prostaglandin E2 and matrix metalloproteinases (MMP), which in turn results in stimulating the osteoclasts with subsequent bone resorption. IL-1 is associated with the erosive processes in the articular cartilage (8). The same cytokines take part in CP. There's a link between the increased serum levels of TNF- $\alpha$ and RA activity (29).

Two studies on anti-TNF- $\alpha$ therapy in RA show that the treatment with them leads to improvement in the condition of the periodontium. This supports the mutual pathogenesis of RA and CP, and the role of TNF$\alpha$ in both diseases $(1,11,35)$. The intake of disease-modifying antirheumatic drugs (DMARDs) improves the condition of the periodontium in patients with RA $(23,36)$. This is in favour of the role of infections and the link between RA and CP too.

What is interesting is that $72 \%$ of the control group show positive anti-P.gingivalis $\mathrm{HtpG}$ antibodies, compared to $63,3 \%$ of the patients with new-onset RA and $50 \%$ of chronic RA (6).

The spread of microorganisms in patients with RA and the control group does not show significant differences (Figure 2). Despite the discovered differences, a single certain bacterial genus, class or family, which should be different in patients with new-onset, chronic RA and the control groups has not been found. The differences are seen mostly when the groups are combined according to the severity of the periodontitis. According to Holt $(37,38)$, the non-pathogenic microflora in the periodontium is dominated by 7 genuses, including Bacteroides (21,3\%), Firmicutes (10,9\%), ctinobacteria (21,8\%), Proteobacteria (16,9\%), Fusobacteria (24\%), Spirochaetes (2,5\%) and TM7 (1,6\%). The transition from moderate to 
severe forms of CP is accompanied by increase of Bacteroides, Spirochaetes and TM7, and decrease of ctinobacteria and Proteobacteria simultaneously (6).

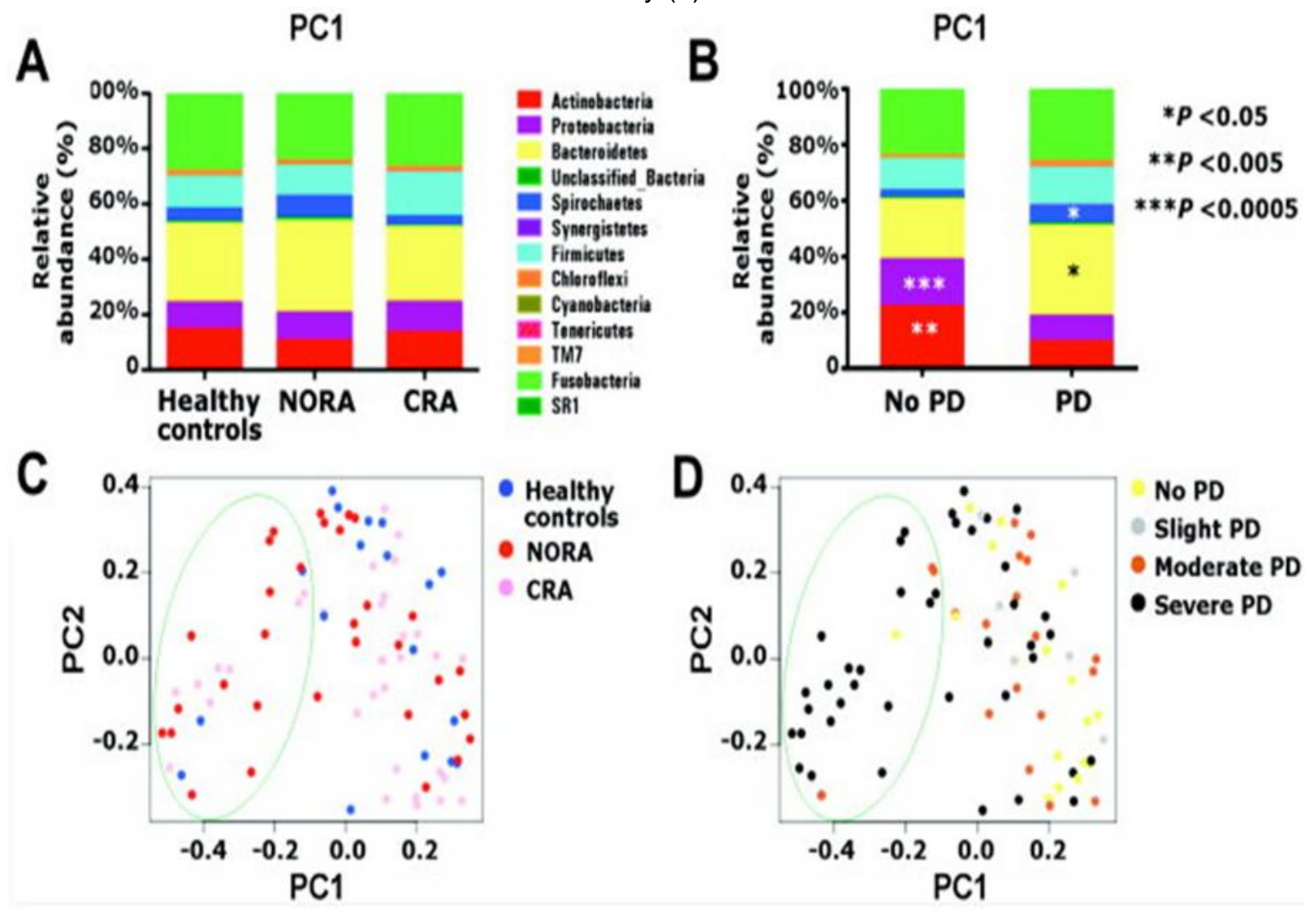

Figure 2. (By Scher et al. Periodontal disease and the oral Microbiota in New-onset Rhematoid Arthritis, 2012)

Figure $2(A)$ shows the distribution of microorganisms in patients with new-onset and chronic $R A$, as well as in a healthy control group (NORA, CRA, Healthy controls).

(B) shows that all patients have been divided into groups according to whether they have or do not have a periodontal disease (No PD, PD). (C) analyses the distribution of patients with new-onset RA compared to those with chronic RA and the control group, compared to graph (D) where grouping is relevant to the severity of CP. It has been estimated that severe forms predominate in patients with new-onset RA (6).

\section{Microorganisms in chronic periodontitis and their potential link to rheumatoid arthritis}

\section{development}

\section{- Porphyromonas gingivalis}

The Porphyromonas genus and P.gingivalis in particular are associated with the severity of $\mathrm{CP}$ and are not specific to the microflora in RA, nor are they associated with the titer of ACPA. Porhyromonas is present in almost all examined patients with a change in its levels (Figure 2). P.gingivalis can be found in $55 \%$ of patients with new-onset RA and in $47 \%$ of patients with chronic RA, whereas the prevalence in 
control groups is $27 \%$. The patients with new-onset RA were divided into 2 subgroups depending on whether there is or isn't CP. P.gingivalis was proved to predominate $(62,5 \%$ against $28,5 \%)$ in patients with advanced CP. Put together these data support the statement that the difference can be explained with greater prevalence of $\mathrm{CP}$ in patients with $\mathrm{RA}$, although the colonisation with P.gingivalis is twice as high in patients with RA compared to the control groups (6).

P.gingivalis is the only microorganism, documented to express PAD (peptidyl arginine deiminase), which takes part in the citrullination process. The expression of citrullinated autoantigens in the synovial fluid supports the role of citrullination in the pathogenesis of RA (27). The citrullinated peptides act as antigens that are ingested by the antigen-presenting cells. They stimulate T-lymphocytes, which secrete cytokines, lymphokines and stimulate B-lymphocytes to produce anti-citrullinated antibodies.

Immunisation with cysteine proteases, purified from $\mathrm{P}$. gingivalis against $\mathrm{CP}$, may be important to the prevention from RA and its treatment (24). P. gingivalis is associated with the citrullination process and might participate in the loss of self-tolerance after the development of RA. The results indicate that the citrullinated peptides are present in the gingiva of patients with CP (31).

The antibodies against P.gingivalis are associated with ACPA in patients with RA (39).

That way, P. gingivalis can be directly associated with RA by the citrullination processes. It's also a proof that the pathogens associated with the severity of CP play a role in RA development and progression (40).

\section{- Porhyromonas, Tannerella and Treponema}

The bacteria in the red complex (triad of the most virulent periodontopathogens - Porhyromonas, Tannerella and Treponema), associated with the severity of $\mathrm{CP}$, predominate in patients with new-onset RA, compared to those with chronic RA (Figure 3 ). It is probably due to the intake of disease-modifying antirheumatic drugs $(6,11)$.
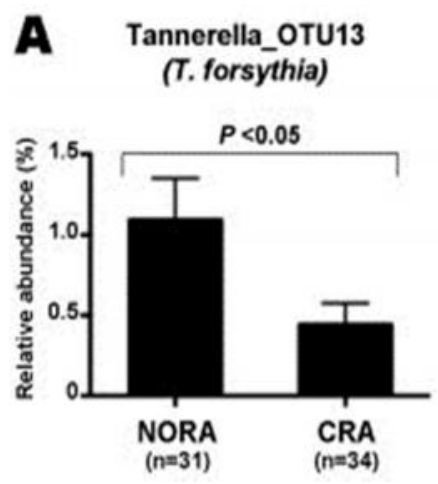

B

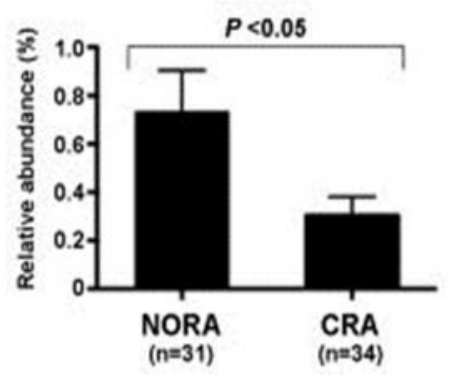

C Porphyromonas_oTU1 ( $P$. gingivalis)

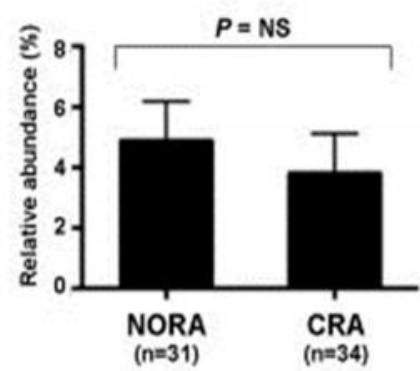

Figure 3. ( By Scher et al. Periodontal disease and the oral Microbiota in New-onset Rhematoid Arthritis, 2012)

Figure 3 shows the distribution of periodontopathogens in patients with new-onset rheumatoid arthritis (NORA) and chronic rheumatoid arthritis (CRA). The microorganisms that are associated with the severity 
of $\mathrm{CP}-(\mathrm{A})$ Tannerella forsythia and (B) Treponema medium are significantly more common in patients with new-onset RA, compared to those with chronic RA. (C) Significant differences in the distribution of Porhyromonas gingivalis in the separate examined groups are not found (6).

\section{- Anaeroglobus geminates and presence of anti-CCP antibodies and/or rheumatoid factor (RF).}

A.geminatus belongs to the Veillonellaceaces family. It is an obligate anaerobe, Gram-negative. The proposed mechanisms that P.gingivalis might use to participate in RA pathogenesis include the ability to produce citrullinated peptides using the peptidylarginine deiminase (PAD) enzyme. It has been estimated that there's no link between RF and CPP, and the presence of Porhyromonas gingivalis in the oral microflora $(20,41)$. Indeed P.gingivalis is not present in some patients with anti-CCP and the titer of antibodies is not positively associated with the bacteria. The presence of Anaeroglobus genus and the OTU99 genus level, significantly relates to the circulating RF and CCP. Besides, OTU99 is associated with CP and can be found in $77,5 \%$ and $50 \%$ of patients with new-onset and chronic RA, but only in $16,7 \%$ of the control groups (6).

\section{- Prevotella and Leptotrichia species - unique specific microorganisms in patients with early rheumatoid arthritis regardless of the severity of chronic periodontitis.}

By applying multivariate statistical analysis (38) it has been estimated that most of the microorganisms that are seen in patients with new-onset RA are not different from the patients with chronic RA and the control group. There is evidence that Prevotella spp and Leptotrichia spp are the only specific microorganisms in patients with new-onset RA regardless of the periodontal status (respectively $32,2 \%$ for Prevotella spp and 25,0\% for Leptotrichia spp) and are not found in the control group $(38,42)$.

\section{Conclusion}

Although the genus Porphyromonas is present in all patients that were examined, its presence is in a direct link to the severity of CP regardless of the rheumatoid arthritis condition. P.gingivalis could be a triggering factor for the development of RA. There are a number of questions that still remain unanswered: if a certain Porphyromonas is responsible for triggering RA, then what explains the occurrence of the disease in patients with no P.gingivalis found? Some of the periodontopathogens (Tannerella,Treponema) are statistically more common in patients with new-onset RA tending to decrease if the disease is wellcontrolled (38). Two types of microorganisms Prevotella spp and Leptotrichia spp are found in patients with new-onset RA only (22). Anaeroglobus geminatus is significantly linked to the titer of RF and CCP. The latter is associated with CP and has been found in patients with new-onset and chronic RA. Prevotella, Leptotrichia and A.geminatus need further investigation and are candidates for target periodontopathogenic bacteria in RA.

The significance of periodontal diseases and the role of periodontopathogenic bacteria in new-onset RA still raise a number of questions that need to be answered.

\section{References}


1. Martinova, F. Clinical significance of HLA immunogenic research in rheumatic diseases, 2004; Doctoral dissertation (in Bulgarian).

2. Dissick, A., Redman, R.S., Jones, M., et al. Association of periodontitis with rheumatoid arthritis: a pilot study. J Periodontol 2010; 81: 223-230.

3. Okda, M., Kobayashi, T., Ito, S., et al. Antibody responses to periodontopathic bacteria in relation to rheumatoid arthritis in Japanese adults. J Periodontol 2011; 82: 1433-1441.

4. Trombone, A.P., Claudino, M., Colavite, P., et al. Periodontitis and arthritis interaction in mice involves a shared hyper-inflammatory genotype and functional immunological interferences. Genes Immun 2010; 11: 479-489.

5. John, N. M. Carranza's Clinical periodontology. (11th ed.), 2012; 160-163.

6. Scher, J. U., Ubeda, C., Equinda, M., et al. Periodontal disease and the oral Microbiota in Newonset Rhematoid Arthritis, 2012; 4: 30-35.

7. Ospelt, C., Reedquist, K.A., Gay, S., et al. Inflammatory memories: is epigenetics the missing link to persistent stromal cell activation in rheumatoid arthritis? Autoimmun Rev 2011; 10: 519-524.

8. нчовск, ., ирков, ., евм тоидный ртрит и хронический п родонтит - хронические з болев ния с общим п тогенезом, учно-пр ктическ я ревм тология, 2007.

9. de Pablo, P., Chapple, I.L., Buckley, C.D., et al. Periodontitis in systemic rheumatic diseases. Nat Rev Rheumatol. 2009; 5: 218-224.

10. Page, R.C., Lantz, M.S., Darveau, R., et al. Immunization of Macaca fascicularis against experimental periodontitis using a vaccine containing cysteine proteases purified from Porphyromonas gingivalis. Oral Microbiol Immunol 2007; 22: 162-168.

11. Elkhouli, A.M. The efficacy of host response modulation therapy (omega-3 plus low0dose aspirin) as an adjunctive treatment of chronic periodontitis (clinical and biochemical study). J Periodontal Res 2011; 46: 261-268.

12. Easlick, K. An Evaluation of the effect of dental focal infection on health. JADA 1952; 42: 609-697.

13. Silman, A.J. and Hochberg, M.C. Epidemiology of the rheumatic diseases, (2nd ed.) New York: Oxford University Press; 2001.

14. Wegner, N., Wait, R., Sroka, A., et al. Peptidylarginine deiminase from Porphyromonas gingivalis citrullinates human fibrinogen and alpha-enolase:implication for autoimmunity in rheumatoid arthritis. Arthritis Rheum. 2010; 62: 2662-2272.

15. Gomez, R.S., Dutra, W.O., Moreira, P.R. Epigenetics and periodontal disease: future perspectives. Inflamm Res 2009; 58: 625-629. 
16. Kinloch, A., Lundberg, K., Wait, R., et al. Synovial fluid is a site of citrullination of autoantigenes in inflammatory arthritis. Arthritis Rheum 2008; 58: 2287-2295.

17. Persson, G.R., Engel, D., Whitney, C., et al. Immunization against Porphyromonas gingivalis inhibits progression of experimental periodontitis in nonhuman primates. Infect immune 1994; 62: 1026-1031.

18. Choi, J., Lee, S.Y., Kim, K., et al. Identification of immunoreactive epitopes of the Porphyromonas gingivalis heat shock protein in periodontitis and atherosclerosis. Periodontal Res 2011; 46: 240245.

19. Hernandez, M., Dutzan, N., Garcia-Sesnich, J., et al. Host-pathogen interactions in progressive chronic periodontitis. J Dent Res 2011; 90: 1164-1170.

20. Seymour, G.J., Ford, P.J., Cullinan, M.P., et al. Relationship between periodontal infections and systemic disease. Clin Microbiol Infect 2007; 13: 3-10.

21. Papapanou PN, Behle JH, Kebshull $\mathrm{M}$ et al. Subgingival bacterial colonization profiles correlate with gingival tissue gene expression. BMC Microbiol 2009; 9: 221.

22. Schafer, A.S., Jepsen, S., Loos, B.G. Periodontal genetics: a decade of genetic association studies mandates better study designs. J Clin Periodontol 2011; 38: 103-107.

23. Rosenstein, E.D., Weismann, G., Greenwald, R.A. Porphyromonas gingivalis, periodontitis and rheumatoid arthritis. Med Hypotheses. 2009; 73: 457-458.

24. Moritz, A.J., Cappelli, D., Lantz, M.S., et al. Immunization with Porphyromonas gingivalis Cysteine protease: effects on experimental gingivitis and ligature-induced periodontitis in Macaca fascicularis. J Periodontol 1998; 69: 686-697.

25. Rosenstein, E.D., Greenwald R.A., Kushner, L.J., et al. Hypothesis: the humoral immune response to oral bacteria provides a stimulus for the development of rheumatoid arthritis. Inflammation 2004; 28: 311-318.

26. Martinez-Martinez, R.E., Abud-Mendoza, C., Patino-Marin, N., et al. Detection of periodontal bacterial DNA in serum and synovial fluid in refractory rheumatoid arthritis patients. J Clin Periodontol 2009; 36: 1004-1010.

27. Lundberg, K., Kinloch, A., Fisher, B.A., et al. Antibodies to citrullinated alpha-enolase peptide 1 are specific for rheumatoid arthritis and cross-react with bacterial enolase. Arthritis Rheum 2008; 58: 3009-3019.

28. Pischon, N., Roehner, E., Hocke, A., et al. Effects of Porphyromonas gingivalis on cell cycle progression and apoptosis of primary human chondrocytes. Ann Rheum Dis 2008; 68: 1902-1907.

29. Persson, G.R. Rheumatoid arthritis and periodontitis - inflammatory and infectious connection. Journal of Oral Microbiology 2012; 4: 25-28. 
30. Lu J, Zhang W, Hao Y: Defect of cell wall construction may shield oral bacteria's survival in bloodstream and cause infective endocarditis. Med Hypotheses 2009, 73:1055-1057

31. Rantapaa-Dahlqvist, S., de Jong, B.A., Berglin, E., et al. Antibodies against cyclic citrullinated peptide and IgA rheumatoid factor predict the development of rheumatoid arthritis. Arthritis Rheum 2003; 48: 2741-2749.

32. Nigrovic, Pa., Lee, D.M. Synovial mast cells: role in acute and chronic arthritis. Immunol Rev 2007; 217: 19-37.

33. Al-Katma, M.K., Bissada, N.F., Bordeaux, J.M., et al. Control of periodontal infection reduces the severity of active rheumatoid arthritis. J Clin Rheumatol 2007; 13: 134-137.

34. Mikuls, T.R., Payne, J.B., Reinhardt, R.A. et al. Antibody responses to Porphyromonas gingivalis in subjects with rheumatoid arthritis and periodontitis. Int Immunopharmacol. 2009; 9: 38-42.

35. Costenbader $\mathrm{KH}$, Prescott J, Zee RY et al. Immunosenescence and rheumatoid arthritis: does telomere shortening predict impending disease? Autoimmun Rev. 2011; 10: 569-573.

36. Sacks, J.J., Luo, Y.H., Helmick, C.G. Prevalence of specific types of arthritis and other rheumatic conditions in the ambulatory health care system in the United States, 2001-2005. Arthritis Care Res 2010; 62: 460-464.

37. Dewhrist FE,Chen T, Izard J, Paster BJ et al. The human oral microbiome. J Bacteriol. 2010; 192: 5002-5017.

38. Holt, S.C., Ebersole, J., Felton, J. et al. Implantation of Bacteroides gingivalis in nonhuman primates initiates progression of periodontitis. Science 1988; 239: 55-57.

39. Hitchon, C.A., Chandal, F., Ferucci, E.D. et al. Antibodies to Porphyromonas gingivalis are associated with anticitrullinated protein antibodies in patient with rheumatoid arthritis and their relatives. J Rheumatol. 2010; 37: 1105-1112.

40. Fredericks, D.N., Relman, D.A. Sequence-based identification of microbial pathogens: a reconsideration of Koch's postulates. Clin Microbiol Rev. 1996; 9: 18-33.

41. Wegner, N., Lundberg, K., Kinloch, A., et al. Autoimmunity to specific citrullinated proteins gives the first clues to the etiology of rheumatoid arthritis. Immunol rev. 2010; 233: 34-54.

42. Eribe, E.R.K., Paster, B.J., Caugant, D.A., et al. Genetic diversity of Leptotrichia and description of Leptotrichia goodfellowii sp. Nov., Leptotrichia hofstadi sp. Nov., Leptotrichia shahii sp. Nov. and Leptotrichia wadei sp. Nov. Int J Syst Evol Microbiol. 2004; 54: 583-92.

\section{Corresponding author:}

Liliya Kavlakova; Department of Periodontology and Oral mucosal diseases, 
Faculty of Dental Medicine, Medical University - Plovdiv, 3 Hristo Botev Blvd, 4000 Plovdiv, Bulgaria; +359 326020 77; Kavlakovi@abv.bg 